\title{
Femoral malrotation after intramedullary nailing in obese versus non-obese patients
}

\author{
John D. Koerner ${ }^{\mathrm{a}}$, Neeraj M. Patel ${ }^{\mathrm{b}}$, Richard S. Yoon ${ }^{\mathrm{b}}$, Mark J. Gage ${ }^{\mathrm{b}}$, Derek J. Donegan ${ }^{\mathrm{c}}$, \\ Frank A. Liporace ${ }^{\mathrm{b}, *}$ \\ a Division of Spine Surgery, Department of Orthopaedic Surgery, Thomas Jefferson University Hospital, Philadelphia, PA, USA \\ ${ }^{\mathrm{b}}$ Division of Orthopaedic Trauma, Department of Orthopaedic Surgery, NYU Hospital for Joint Diseases, New York, NY, USA \\ ${ }^{c}$ Division of Orthopaedic Trauma, Department of Orthopaedic Trauma, Hospital of the University of Pennsylvania, Philadelphia, PA, USA
}

A R T I C L E I N F O

\section{Article history:}

Accepted 2 February 2014

\section{Keywords:}

BMI

Obesity

Intramedullary nail

Femoral version

Outcomes level of evidence: Level III

prognostic

\begin{abstract}
A B S T R A C T
Objective: Intramedullary nailing (IMN) of obese patients with femoral fractures can be difficult due to soft tissue considerations and overall body habitus. Complications including malrotation can occur and have significant impact on postoperative function. The purpose of this study was to evaluate femoral rotation after intramedullary nailing of obese and non-obese patients to see if there was a difference in rotation, complications and any risk factors for malrotation.

Materials and methods: Between 2000 and 2009, 417 consecutive patients with femur fractures treated with IM nail at Level I trauma and tertiary referral center. Of these, 335 with postoperative computed tomography (CT) scanogram of the bilateral lower extremities were included in this study. Baseline demographic, perioperative and postoperative femoral version calculations were included in the dataset. Statistical analysis included chi-squared test for categorical data, $t$-test for continuous data, and univariate and multivariate regression analysis. Significance was set at $p<0.05$.

Results: Of the 417 patients with femur fractures between 2000 and 2009, 335 met criteria for this study. There were 111 patients with a BMI $<25,129$ with BMI 25-29.9, and 95 patients with a BMI $>30$. When BMI was categorised into 3 groups $(<25,25-29.9$, or $30+)$, none of these groups were predictive of version in univariate or multivariate regressions. Among only obese patients (BMI 30+), BMI of 35+ was not a significant predictor of version when compared to BMI 30-34.9. There were no significant differences in femoral version based on entry point (antegrade vs. retrograde) in any BMI category. There were also no significant difference between groups of patients with a DFV of $>15^{\circ}(p=0.212)$.

Conclusions: Based on this study, BMI did not have an effect on postoperative difference in femoral version. In fact, in our multivariate regression analysis, BMI of over 30 was actually predictive of significantly lower difference in femoral version. While other studies have documented the intraoperative difficulties encountered with obese patients with femur fractures, the outcome of femoral rotation is not affected by an increasing BMI.
\end{abstract}

(c) 2014 Elsevier Ltd. All rights reserved.

\section{Introduction}

According to the National Health and Nutrition Examination Survey, $37.5 \%$ of U.S. adults were obese in 2009-2010 [1], which has increased from $27.5 \%$ in $1999-2000$ [2]. The health risks associated with obesity including hypertension, increased lipid concentrations, and type 2 diabetes have been well documented [1]. The complications in obese patients undergoing orthopaedic

\footnotetext{
* Corresponding author. Tel.: +1 201309 2426; fax: +1 2125987654

E-mail address: liporace33@gmail.com (F.A. Liporace).
}

spine and arthroplasty surgery have also been well documented [3-7].

Some of the difficulties encountered in femoral nailing of obese patients have been described [8-10], and have led to obesity being a relative indication for retrograde nailing [8,11-13]. Tucker et al. prospectively analysed obese patients undergoing femoral nailing, and found significantly shorter operative time, and less fluoroscopy usage in retrograde versus antegrade nailing [8]. Based on postoperative radiographs, no significant differences were found for alignment, as well as no significant differences in leg lengths and rotational deformity, which was measured clinically. 
Malrotation following intramedullary nailing (IM) of femur fractures may lead to complications such as malunion, alteration of gait and patellofemoral mechanics, limitation of hip motion, and ultimately lead to patient dissatisfaction. The rate of malrotation following IM nailing may be as high as $27.6 \%$ [14-18]. Intraoperative methods for assessing rotation include lesser trochanter profile, cortical thickness, as well as clinical comparison to the opposite side have been described [19-21]. These methods to assess rotation may become more inaccurate in patients that are obese, as radiographs become more difficult to read, and body habitus limits the ability to compare to the contralateral extremity. Prior studies have retrospectively reviewed rotation after femur IM nailing in regards to multiple factors, but did not examine obesity as a potential factor [22]. To our knowledge, there are no studies looking at rotational malalignment after femoral IM nailing in obese patients, specifically comparing antegrade versus retrograde.

\section{Materials and methods}

Between 2000 and 2009, 417 consecutive patients with femoral diaphyseal fractures were treated with an IM nail and included retrospectively in our institution's femoral IM nail registry. Inclusion criteria for this study included complete baseline and demographic documentation as well as postoperative CT scanogram for version measurement of the bilateral femurs. Exclusion criteria included bilateral injuries, previous injury, previous deformity, and those with complete data sets. Of the initial 417 subjects, 335 met the inclusion criteria and were included in this study. Institutional protocol dictated that one of the two following methods was used to determine rotation for all fractures in which it could not be assessed intra-operatively by cortical alignment due to comminution: (1) pre-operatively, the contour of the lesser trochanter was noted fluoroscopically with the ipsilateral patella facing directly anterior. This saved image was then used in order to match a similar lesser trochanteric contour on the injured femur. or (2) the quantitative measurement test published by Tornetta et al. [21]. Institutional protocol during this time period also dictated a postoperative CT scanogram to assess femoral version. This was voted by the faculty to make protocol and was outside the realm of IRB approval; however, to utilise the data, IRB approval was obtained prior to study start.

Femoral version was measured bilaterally as originally described by Dugdale et al. [23] CT scanogram was used to obtain axial images through the proximal and distal femurs. At the hip, a line was drawn along the axis of the femoral neck and referenced to the horizontal axis. A second line was then drawn tangential to the posterior femoral condyles and also referenced to the horizontal axis. These angles were then used to calculate the femoral version of the extremities. The difference in version between the unaffected (normal) extremity and the injured extremity was defined at difference in femoral version (DFV). A fellowshiptrained orthopaedic trauma surgeon measured version on each CT scanogram.

\section{Statistical methods}

BMI was first treated as a continuous variable. It was then reclassified into a categorical variable as follows: BMI $<25$, BMI $25-$ 29.9, and BMI 30+. Per group, mean DFV was calculated and compared without controlled regression analysis via ANOVA. In addition to BMI, we also sought to evaluate any potential effect of body weight. Other data included in our analysis were age, sex, mechanism of injury, open vs. closed fracture, antegrade vs. retrograde IMN, nail starting point (greater trochanter vs. piriformis), and $\mathrm{AO}$ and Winquist classifications. Standard descriptive statistics, including mean and standard deviation (SD), were used to report baseline and demographic data. Categorical data were analysed using chi-square or Fisher exact tests, as appropriate. Next, including all of the aforementioned variables, regression analysis was conducted. First, univariate, followed by stepwise, multivariate regressions were used to test for associations between independent variables and our primary outcome variable (DFV). Statistical significance was defined as $p<0.05$. All statistical analysis was performed with SAS 9.0 (SAS Institute Inc., Cary, NC). Finally, post-hoc power analysis yielded that at an alpha of 0.05 and power of $0.80,92$ patients would be required in each group to achieve statistical significance. To achieve a power of $0.90,129$ patients would be required in each group, leaving the power of our study in between 0.80 and 0.90 .

\section{Results}

Of the 417 patients with femur fractures between 2000 and 2009,335 met criteria for this study. There were 111 patients with a BMI $<25$ (33.1\%), 129 with BMI 25-29.9 (38.5\%), and 95 patients with a BMI $>30(28.4 \%)$. As a continuous variable, BMI was significantly, albeit weakly, predictive of post-operative DFV in univariate regression $\left(p=0.038, R^{2}=0.013\right)$. However, statistical significance was lost in stepwise, multivariate regression when accounting for age, sex, mechanism of injury, open vs. closed fracture, nail insertion method and start point, and AO and Winquist classifications.

The mean DFV for each of the three aforementioned BMI categories is listed in Table 1. Among only obese patients (BMI $30+$ ), BMI of $35+$ was not a significant predictor of version when compared to BMI 30-34.9. Body weight was not a predictor of DFV either in univariate or multivariate analysis. There were no significant differences in mean femoral version based on antegrade versus retrograde nailing or when comparing piriformis versus trochanteric start points between the three BMI categories (Tables 2 and 3, respectively). Furthermore, there were no statistically significant differences in mean DFV when comparing "low grade" (types 1 and 2) and "high grade" (types 3 and 4) Winquist classifications among the three groups (Table 4). Subsequently, none of the three BMI categories were predictive of post-operative DFV when controlling for independent factors in a multivariate model. There was also no significant difference between groups in the proportion of patients with a DFV of $>15$ (Table 5 ).

\section{Discussion}

More than one out of three people in the U.S. are obese, and treating obese patients with femur fractures has many implications. In addition to the increased costs of treating obese patients associated with longer rehabilitation times [24], there are technical considerations during the procedure that may alter outcomes. Potential difficulties with starting point and guide wire placement have lead some to advocate for retrograde nails in obese patients [8-10].

Difficulties encountered during antegrade femoral nailing of obese patients has been documented by multiple operating room

Table 1

Difference in femoral version by BMI grouping.

\begin{tabular}{lllll}
\hline & BMI $<25$ & BMI 25-29.9 & BMI 30+ & $p$ Value \\
\hline Total $n$ & 111 & 129 & 95 & - \\
Mean DFV (SD) & $9.5(7.24)$ & $9.8(7.69)$ & $7.3(6.7)$ & $\mathbf{0 . 0 0 6}^{*}$ \\
\hline
\end{tabular}

Group comparison via ANOVA, sig $p<0.05$

$\mathrm{BMI}=$ body mass index; DFV = difference in femoral version. 
Table 2

Difference in femoral version by BMI and entry point.

\begin{tabular}{lllll}
\hline Grouping & $n$ & Antegrade DFV (SD) & $n$ & $p$ Retrograde DFV (SD) \\
\hline BMI $<25$ & 81 & $9.7(7.36)$ & 30 & $8.9(7.0)$ \\
BMI 25-29.9 & 80 & $9.5(7.34)$ & 49 & $10.49(8.35)$ \\
BMI 30+ & 48 & $6.47(5.95)$ & 47 & 0.61 \\
\hline
\end{tabular}

"Group comparison via $t$-test, sig $p<0.05$.

$\mathrm{BMI}=$ body mass index; $\mathrm{DFV}=$ difference in femoral version

Table 3

Difference in femoral version by BMI and antegrade starting point.

\begin{tabular}{lllll}
\hline Grouping & $n$ & Piriformis DFV (SD) & $n$ & Trochanteric DFV (SD) \\
\hline BMI $<25$ & 54 & $9.7(7.82)$ & 27 & $9.9(6.44)$ \\
BMI 25-29.9 & 58 & $9.8(7.91)$ & 22 & $9.4(7.44)$ \\
BMI 30+ & 27 & $6.6(6.79)$ & 21 & $6.1(4.87)$ \\
\hline
\end{tabular}

Table 4

Difference in femoral version by BMI and Winquist (low grades $=1 / 2$; high grades $=3 / 4$ ).

\begin{tabular}{lllll}
\hline Grouping & $n$ & Low grade DFV (SD) & $n$ & High grade DFV (SD) \\
\hline BMI $<25$ & 75 & $9.2(7.28)$ & 36 & $9.9(6.15)$ \\
BMI 25-29.9 & 87 & $9.9(7.70)$ & 42 & 0.62 \\
BMI 30+ & 54 & $6.3(5.08)$ & 41 & 0.84 \\
\hline
\end{tabular}

Table 5

Percentage of patients with DFV $>15$ by BMI category.

\begin{tabular}{lll}
\hline Grouping & $n$ With DFV $>15 \%(\%)$ & $p$ Value \\
\hline BMI $<25$ & $21 / 111(18.92)$ & 0.21 \\
BMI 25-29.9 & $26 / 129(20.16)$ & \\
BMI 30+ & $11 / 95(11.58)$ & \\
${ }^{*}$ Group comparison via chi-square, sig $p<0.05$. & \\
BMI = body mass index; DFV = difference in femoral version.
\end{tabular}

variables, as well as complications. Tucker et al. found a $52 \%$ greater average operative time in obese patients during antegrade nailing, but no difference in average operative time in retrograde nailing [8]. The authors also found a $79 \%$ greater average fluoroscopy time in obese patients undergoing antegrade nailing versus non obese patients. In the morbidly obese population, McKee also observed longer operative time, as well as increased blood loss when compared to non-morbidly obese patients [9]. While obese patients may provide challenges within the operating room, little data exists on how this will affect their outcome. Complication rates have been found to be similar in obese and non obese patients following IM nail in regards to delayed union, nonunion, malunion, and infection $[8,25]$. Comparing version in obese and non obese patients after femoral nailing may help us in treating these patients, as well as deciding which entry point to use.

Based on this study, BMI did not have an effect on postoperative difference in femoral version. In fact, in our multivariate regression analysis, BMI of over 30 was actually predictive of significantly lower difference in femoral version. While other studies have documented the intraoperative difficulties encountered with obese patients with femur fractures, the outcome of femoral rotation is not affected by an increasing BMI. One possible explanation for this finding, is that having a larger BMI and soft tissue envelope around the femur, will potentially decrease the amount of initial displacement and allow for a more anatomic reduction. In addition, the surgical team may be more aware of the potential difficulties when faced with an obese patient, and be more diligent in attempting to prevent malrotation. Also, intraoperative clinical assessment of limb alignment may be more difficult in obese patients, and relying more on radiographic criteria of alignment may be more accurate.

\section{Conflict of interest statement}

The authors have no direct or indirect conflict of interests and have no received funding in regards to this project. Ethical review board approval was obtained prior to the start and maintained until completion of the study.

\section{References}

[1] Ogden CL, Carroll MD, Kit BK, et al. Prevalence of obesity in the United States, 2009-2010. NCHS Data Brief 2012;82:1-8.

[2] Ogden CL, Carrol MD, Curtin LR, et al. Prevalence of overweight and obesity in the United States, 1999-2004. JAMA 2006;295(13):1549-55.

[3] Winiarsky R, Barth P, Lotke P. Total knee arthroplasty in morbidly obese patients. J Bone Joint Surg Am 1998;80A:1770-4.

[4] Olsen MA. Risk factors for surgical site infection in spinal surgery. J Neurosurg Spine 2003;98:149-55.

[5] Foran JRH, Mont MA, Etienne G, et al. The outcome of total knee arthroplasty on obese patients. J Bone Joint Surg Am 2004;86A:1609-15.

[6] Jerabek S, Karunakar MA, Shah SN. Body mass index as a predictor of complications after operative treatment of acetabular fractures. J Bone Joint Surg Am 2005;87A:1498-502.

[7] Colizza W, Griffin FM, Insall NJ, et al. Total knee arthroplasty in patients who were obese with 10 years followup. Clin Orthop Relat Res 1998;356:28-33.

[8] Tucker MC, Schwappach JR, Leighton RK, et al. Results of femoral intramedullary nailing in patients who are obese versus those who are not obese: a prospective multicenter comparison study. J Orthop Trauma 2007;21:523-9.

[9] McKee MD, Waddell JP. Intramedullary nailing of femoral fractures in morbidly obese patients. J Orthop Trauma 1994;36:208-10.

[10] Ostrum RF. A greater trochanteric insertion site for femoral nailing in lipomatous patients. Orthopaedics 1996;19:337-40.

[11] Anup K, Mehra MM. Retrograde femoral interlocking nail in complex fractures. J Orthop Trauma 2002;10:17-21.

[12] Ricci WM, Bellabarba C, Evanoff B, et al. Retrograde versus antegrade nailing of femoral shaft fractures. J Orthop Trauma 2001;15:161-9.

[13] Herscovici Jr D, Whiteman KW. Retrograde nailing of the femur using an intercondylar approach. Clin Orthop 1996;332:98-104.

[14] Winquist RA, Hansen Jr ST, Clawson DK. Closed intramedullary nailing of femoral fractures. A report of five hundred and twenty cases. J Bone Joint Surg Am 1984;66:529-39.

[15] Braten M, Terjesen T, Rossvoll I. Femoral anteversion in normal adults. Ultrasound measurements in 50 men and 50 women. Acta Orthop Scand 1992;63:29-32. 
[16] Yang KH, Han DY, Jahng JS, et al. Prevention of malrotation deformity in femoral shaft fracture. J Orthop Trauma 1998;12:558-62.

[17] Stephen DJ, Kreder HJ, Schemitsch EH, et al. Femoral intramedullary nailing: comparison of fracture-table and manual traction. a prospective, randomized study. J Bone Joint Surg Am 2002;84:1514-21.

[18] Salem KH, Maier D, Keppler P, et al. Limb malalignment and functional outcome after antegrade versus retrograde intramedullary nailing in dista femoral fractures. J Trauma 2006;61:375-81.

[19] Langer JS, Gardner MJ, Ricci WM. The cortical step sign as a tool for assessing and correcting rotational deformity in femoral shaft fractures. J Orthop Trauma 2010;24:82-8.

[20] Jaarsma RI, van Kampen A. Rotational malalignment after fractures of the femur. J Bone Joint Surg Br 2004;86:1100-4
[21] Tornetta III P, Ritz C, Kantor A. Femoral torsion after interlocked nailing of unstable femoral fractures. J Trauma 1995;38:213-9.

[22] Hufner T, Citak M, Suero EM, et al. Femoral malrotation after unreamed intramedullary nailing: an evaluation of influencing operative factors. J Orthop Trauma 2011;25:224-7.

[23] Dugdale TW, Degnan GG, Turen $\mathrm{CH}$. The use of computed tomographic scan to assess femoral malrotation after intramedullary nailing: A case report. Clin Orthop Relat Res 1992;279:258-63.

[24] Baldwin KD, Matuszewski PE, Namdari S, Esterhai JL, Mehta S. Does morbid obesity negatively affect the hospital course of patients undergoing treatment of closed, lower-extremity diaphyseal long-bone fractures? Orthopedics 2011;34(1):18.

[25] Wolinsky PR, McCarty E, Shyr Y, et al. Reamed intramedullary nailing of the femur: 551 cases. J Trauma 1999;46(3):392-9. 